\title{
Genomic Alterations and Outcomes with VEGF-Targeted Therapy in Patients with Clear Cell Renal Cell Carcinoma
}

\author{
M.I. Carlo ${ }^{\mathrm{a}}$, B. Manley ${ }^{\mathrm{b}}$, S. Patil ${ }^{\mathrm{c}}$, K.M. Woo $^{\mathrm{c}}$, D.T. Coskey ${ }^{\mathrm{a}}$, A. Redzematovic ${ }^{\mathrm{a}}$, M. Arcila ${ }^{\mathrm{d}}$, \\ M. Ladanyid ${ }^{d}$, W. Lee ${ }^{\mathrm{e}}$, Y.B. Chen ${ }^{\mathrm{d}}$, C.H. Lee ${ }^{\mathrm{a}}$, D.R. Feldman ${ }^{\mathrm{a}}$, A.A. Hakimi ${ }^{\mathrm{b}}$, R.J. Motzer ${ }^{\mathrm{a}}$, \\ J.J. Hsieh ${ }^{\mathrm{a}, \mathrm{e}}$ and M.H. Voss ${ }^{\mathrm{a}, *}$ \\ ${ }^{a}$ Department of Medicine, Memorial Sloan Kettering Cancer Center, New York, NY, USA \\ ${ }^{\mathrm{b}}$ Department of Surgery, Memorial Sloan Kettering Cancer Center, New York, NY, USA \\ ${ }^{\mathrm{c}}$ Department of Epidemiology/Biostatistics, Memorial Sloan Kettering Cancer Center, New York, NY, USA \\ ${ }^{\mathrm{d}}$ Department of Pathology, Memorial Sloan Kettering Cancer Center, New York, NY, USA \\ ${ }^{\mathrm{e}}$ Department of Human Oncology and Pathogenesis Program, Memorial Sloan Kettering Cancer Center, \\ New York, NY, USA
}

\begin{abstract}
.
Background: Mutations in VHL, PBRM1, SETD2, BAP1, and KDM5C are common in clear cell renal cell carcinoma (ccRCC), and presence of certain mutations has been associated with outcomes in patients with non-metastatic disease. Limited information is available regarding the correlation between genomic alterations and outcomes in patients with metastatic disease, including response to VEGF-targeted therapy.

Objective: To explore correlations between mutational profiles and cancer-specific outcomes, including response to standard VEGF-targeted agents, in patients with metastatic cc RCC.

Methods: A retrospective review of 105 patients with metastatic ccRCC who had received systemic therapy and had targeted next-generation sequencing of tumors was conducted. Genomic alterations were correlated to outcomes, including overall survival and time to treatment failure to VEGF-targeted therapy.

Results: The most frequent mutations were detected in VHL (83\%), PBRM1 (51\%), SETD2 (35\%), BAP1 (24\%), KDM5C (16\%), and TERT (14\%). Time to treatment failure with VEGF-targeted therapy differed significantly by PBRM1 mutation status ( $p=0.01$, median 12.0 months for MT versus 6.9 months for WT) and BAP1 mutation status $(p=0.01$, median 6.4 months for MT versus 11.0 months for WT). Shorter overall survival was associated with TERT mutations $(p=0.03$, median 29.6 months for MT versus 52.6 months for WT) or BAP1 mutations ( $p=0.02$, median 28.7 months for MT versus not reached for WT).

Conclusions: Genomic alterations in ccRCC tumors have prognostic implications in patients with metastatic disease. BAP1 and TERT promoter mutations may be present in higher frequency than previously thought, and based on this data, deserve further study for their association with poor prognosis.
\end{abstract}

Keywords: Clear cell renal cell carcinoma, VEGF-targeted therapy, genomics, prognosis

\footnotetext{
*Correspondence to: Martin Voss, Genitourinary Oncology Service, Department of Medicine, Memorial Sloan Kettering Cancer Center, 1275 York Avenue, New York, NY 10065, USA.
}

Tel.: +1 646422 4631; Fax: +1 646227 2417; E-mail: vossm@ mskcc.org. 


\section{KEY MESSAGE}

The most frequent mutations in metastatic clear cell renal cell carcinoma (ccRCC) are similar to those in localized ccRCC. Certain genetic alterations, such as $B A P 1, P B R M 1$ and $T E R T$ promoter mutations, may have prognostic implications in metastatic ccRCC and should be further investigated.

\section{INTRODUCTION}

Clear cell renal cell carcinoma (ccRCC) is the most common subtype of kidney cancer, estimated to account for about $80 \%$ of the nearly 62,700 cases diagnosed annually in the United States $[1,2]$. The majority of patients are cured by surgical resection, but a considerable portion is either diagnosed with metastatic disease or develops recurrence after surgery. Once the disease is metastatic, it is usually incurable with limited overall survival (OS) [2].

Over the last decade, approved therapies for metastatic ccRCC have expanded considerably. The growing number of available agents includes vascular-endothelial growth factor (VEGF)-directed compounds, mammalian target of rapamycin (mTOR) inhibitors, and recently, checkpoint inhibitors [3-7]. Lack of predictive biomarkers, however, prevents rational sequencing strategies for these regimens. This stands in contrast to other malignancies such as non-small cell lung cancer or melanoma, where validated molecular markers predict response to approved targeted agents [8-10].

Large scale sequencing efforts have helped unveil the genomic landscape of ccRCC $[11,12]$. In addition to confirming $V H L$ alterations as linchpin events in the pathogenesis of ccRCC, they uncovered other recurrent genomic events, including alterations in the tumor suppressors PBRM1, SETD2, BAP1 and $K D M 5 C$. These analyses, however, were mostly carried out in patients with early stage disease. Similarly, prior reports associating some of these genomic alterations with outcome were limited to non-metastatic ccRCC [13-15].

For patients with metastatic disease, key questions remain unanswered, such as the incidence of genomic alterations and the association of alterations with clinical outcomes, including response to targeted therapies. In this study, we retrospectively analyzed patients with metastatic ccRCC treated with systemic therapy that had undergone targeted exome sequencing of cancer-related genes. The aim was to explore correlations between mutational profiles and cancerspecific outcomes, including response to standard VEGF-targeted agents.

\section{METHODS}

\section{Patients and samples}

Patients were identified from an institutional database of RCC patients who had previously undergone next-generation targeted exome sequencing of archival tumor tissue. Subjects with metastatic ccRCC who had received at least one line of systemic therapy at Memorial Sloan Kettering Cancer Center (MSKCC) were included in the analysis. Demographics, clinical characteristics and details on treatment effect were collected for each case via retrospective review of clinical records. This study was approved by the MSKCC Institutional Review Board, and all patients consented to a tissue procurement protocol.

\section{Tissue acquisition and next-generation sequencing (NGS) analysis}

Formalin-fixed, paraffin-embedded (FFPE) tissue specimens from either the primary or a metastatic site were reviewed by genitourinary pathologists to select areas of maximum tumor content for DNA extraction; blood samples were used for matched comparison of germline DNA. For NGS analysis, the MSKCC IMPACT TM (Integrated Mutation Profiling of Actionable Cancer Targets) assay was employed as previously described [16]. IMPACT is designed to detect SNVs, short indels, copy number aberrations and structural rearrangements. NGS was performed using Illumina HiSeq, with deep coverage across all exonic sequences, plus select introns deemed relevant for fusion events. Variants are annotated with Annovar, then further filtered using several criteria, including previous literature, and technical characteristics of the variant call such as depth of coverage. Initial samples (20\%) had 341 genes investigated per an earlier version of the assay, while 410 genes were tested for all subsequent samples (full gene list in Supplemental Table 1, both versions include all commonly mutated genes in ccRCC). Five samples underwent whole genome sequencing.

\section{Statistical analysis}

The relationship between mutation status and overall survival (OS) and time to treatment failure (TTF) 
from treatment initiation of first therapy was evaluated using time to event methods. For OS, patients still alive at the time of analysis were censored at the last clinic date on which survival status could be confirmed. For TTF, an event was considered to be the time of treatment discontinuation, for any reason (progression or toxicity). Patients still on therapy at the time of analysis were censored at that point in time. Kaplan-Meier estimates for both endpoints were summarized by calculating the median with 95\% confidence intervals and for OS 2 year estimates. Comparisons by each mutation were tested using the Log-Rank statistic. Our analyses are hypothesis generating and as such, we do not adjust for multiple comparisons. All analyses were performed in SAS v 9.4 and $\mathrm{R} v$ 3.1.1.

\section{RESULTS}

\section{Patient characteristics}

A total of 105 patients were identified; patient characteristics are summarized in Table 1. Median age was 57 years (range 33-81). MSKCC Risk Group was available for 89 patients starting first-line therapy (35\% favorable, $52 \%$ intermediate and $14 \%$ poor risk). Thirty-six (34\%) patients had received 1 line of therapy at time of analysis, 49 (47\%) had received 2-3 lines, and $20(19 \%)$ had received 4 or more lines.

\section{Mutation analysis}

Mutation frequencies detected in our analysis of 105 tumors (66\% primary tumors, 34\% metastatic lesions) are outlined in Fig. 1. Mean depth of coverage across the cohort was 608x (range 147-1028). The majority of samples $(90.5 \%)$ were collected prior to initiation of treatment with VEGF-targeted agents. The most frequent mutations were VHL (83\%), PBRM1 (51\%), SETD2 (35\%), BAP1 (24\%), KDM5C (16\%), and TERT (14\%). KDM5C and BAP1 mutations were mutually exclusive, while 15 of 17 tumors with $K D M 5 C$ alterations also harbored mutations in PBRM1. Only one tumor had mutations in both $B A P 1$ and TERT (Fig. 1). All but one of the TERT mutations were in the promoter region. When ranking mutation frequencies separately from primary tumors vs. metastatic sites, the top 4 mutated genes (VHL, PBRM1, SETD2, BAP1) remained the same (Supplemental Table 2).
Table 1

Patient characteristics $(\mathrm{N}=105)$

\begin{tabular}{|c|c|}
\hline Age at diagnosis (median, range) & $57(33-81)$ \\
\hline \multicolumn{2}{|l|}{$\operatorname{Sex}$} \\
\hline $\mathrm{F}$ & $28(26.7 \%)$ \\
\hline M & $77(73.3 \%)$ \\
\hline \multicolumn{2}{|l|}{ Nephrectomy } \\
\hline Yes & $95(90.5 \%)$ \\
\hline No & $10(9.5 \%)$ \\
\hline \multicolumn{2}{|l|}{ Type of Nephrectomy } \\
\hline Localized Disease & $44(46.3 \%)$ \\
\hline Cytoreductive & $51(53.7 \%)$ \\
\hline \multicolumn{2}{|l|}{ Sarcomatoid Features } \\
\hline Yes & $30(28.6 \%)$ \\
\hline No & $65(61.9 \%)$ \\
\hline NA & $10(9.5 \%)$ \\
\hline \multicolumn{2}{|l|}{ Grade } \\
\hline G1 & $0(0.0 \%)$ \\
\hline $\mathrm{G} 2$ & $14(13.3 \%)$ \\
\hline G3 & $37(35.2 \%)$ \\
\hline G4 & $45(42.9 \%)$ \\
\hline NA & $9(8.6 \%)$ \\
\hline \multicolumn{2}{|l|}{ Stage at Time of Diagnosis } \\
\hline $\mathrm{I}$ & $11(10.5 \%)$ \\
\hline II & $10(9.5 \%)$ \\
\hline III & $26(24.8 \%)$ \\
\hline IV & $57(54.3 \%)$ \\
\hline NA & $1(1.0 \%)$ \\
\hline \multicolumn{2}{|l|}{ MSKCC Risk Group } \\
\hline Favorable & $31(34.8 \%)$ \\
\hline Intermediate & $46(51.7 \%)$ \\
\hline Poor & $12(13.5 \%)$ \\
\hline NA & $16(18.0 \%)$ \\
\hline \multicolumn{2}{|l|}{ Received a VEGF-Targeted Agent } \\
\hline Yes & $95(90.5 \%)$ \\
\hline No & $10(9.5 \%)$ \\
\hline \multicolumn{2}{|c|}{ Therapies Prior to VEGF-Targeted Agent } \\
\hline None & $94(89.5 \%)$ \\
\hline mTOR inhibitor & $6(5.7 \%)$ \\
\hline Interferon & $5(4.8 \%)$ \\
\hline \multicolumn{2}{|l|}{ First VEGF-Targeted Agent } \\
\hline Sunitinib & $60(57.2 \%)$ \\
\hline Pazopanib & $31(29.5 \%)$ \\
\hline Bevacizumab & $1(1.0 \%)$ \\
\hline Axitinib & $0(0.0 \%)$ \\
\hline Sorafenib & $1(1.0 \%)$ \\
\hline Sunitinib/bevacizumab ${ }^{a}$ & $2(1.9 \%)$ \\
\hline
\end{tabular}

\section{Mutation status and OS}

With a median follow-up of 26 months, median OS from the initiation of treatment for the entire cohort was 50 months (95\% CI 36.3 - not reached). Correlation of mutation status in individual genes of interest with OS is summarized in Table 2. Patients with TERT mutant (MT) tumors had a significantly shorter OS compared to TERT wild-type (WT) tumors (median 29.6 vs. 52.6 months; $p=0.03$ ). Similar adverse 


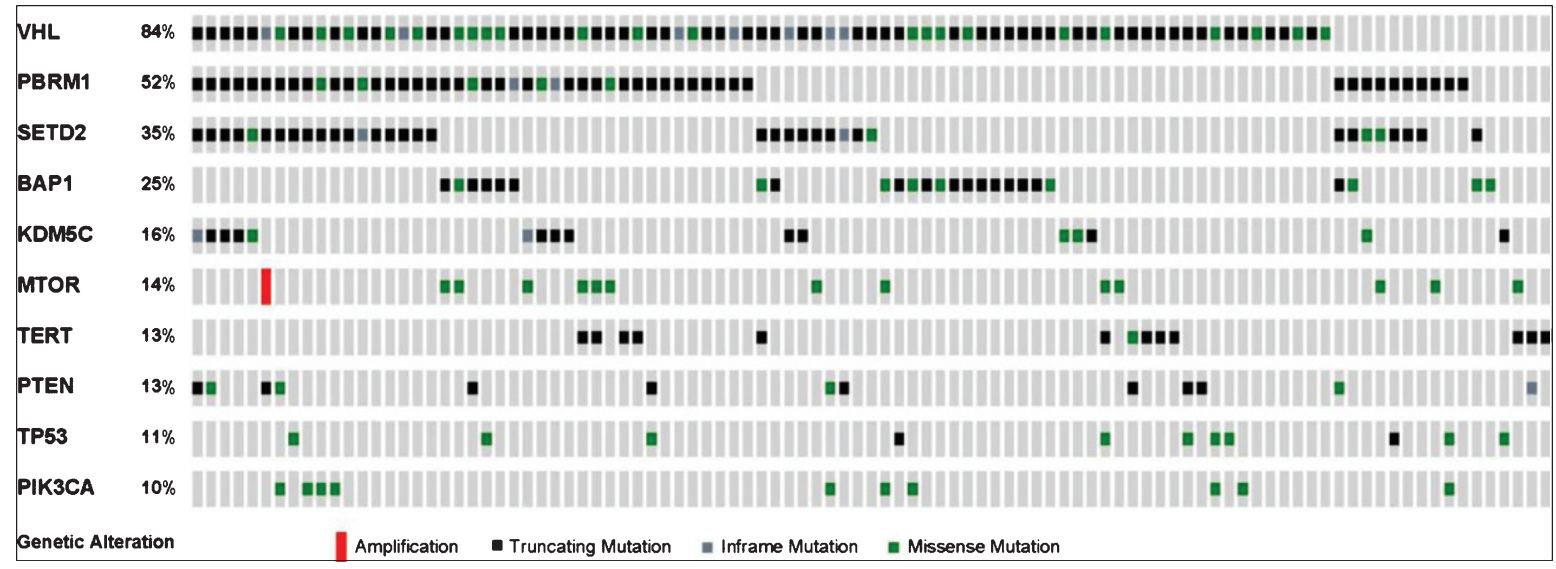

*5 patients with whole genome sequencing were excluded

Fig. 1. Frequency of genetic alterations.

Table 2

Overall survival (OS) by mutation status

\begin{tabular}{lcccccc}
\hline $\begin{array}{l}\text { Mutation } \\
\text { status }\end{array}$ & $N$ & $\begin{array}{c}\text { Median OS } \\
\text { (months) }\end{array}$ & $95 \%$ CI & $\begin{array}{c}\text { Log-rank } \\
p \text {-value }\end{array}$ & $\begin{array}{c}2 \text { year } \\
\text { OS }\end{array}$ & $95 \%$ CI \\
\hline$V H L$ WT & 18 & 26.1 & $(18.4-N A)$ & 0.13 & $62.0 \%$ & $(37-100)$ \\
$V H L$ MT & 87 & 49.9 & $(37.3-\mathrm{NA})$ & & $77.0 \%$ & $(67-87)$ \\
PBRM1 WT & 52 & 36.3 & $(29.6-\mathrm{NA})$ & 0.12 & $72.8 \%$ & $(61-88)$ \\
PBRM1 MT & 53 & $\mathrm{NR}$ & $(49.9-\mathrm{NA})$ & & $77.6 \%$ & $(66-92)$ \\
SETD2 WT & 68 & 40.0 & $(29.6-\mathrm{NA})$ & 0.29 & $72.7 \%$ & $(62-86)$ \\
SETD2 MT & 37 & $\mathrm{NR}$ & $(37.3-\mathrm{NA})$ & & $79.3 \%$ & $(66-96)$ \\
BAP1 WT & 80 & $\mathrm{NR}$ & $(41.2-\mathrm{NA})$ & 0.02 & $78.0 \%$ & $(68-89)$ \\
BAP1 MT & 25 & 28.7 & $(23.9-\mathrm{NA})$ & & $65.4 \%$ & $(48-90)$ \\
KDM5C WT & 88 & 40 & $(32.3-\mathrm{NA})$ & 0.15 & $73.4 \%$ & $(64-85)$ \\
KDM5C MT & 17 & $\mathrm{NR}$ & $(41.2-\mathrm{NA})$ & & $84.0 \%$ & $(66-100)$ \\
TERT WT & 90 & 52.6 & $(40.0-\mathrm{NA})$ & 0.03 & $76.1 \%$ & $(67-87)$ \\
TERT MT & 15 & 29.6 & $(18.4-\mathrm{NA})$ & & $68.6 \%$ & $(46-100)$ \\
\hline
\end{tabular}

*MT, mutant; WT, wild-type; NA, not applicable.

association with outcome was apparent for mutation status in BAPl ( $p=0.02$, median 28.7 months vs. not reached) (Fig. 2). There were no statistically significant OS differences when comparing MT vs. WT for VHL, PBRM1, SETD2, and KDM5C (Table 2).

\section{Mutation status and TTF to VEGF-targeted therapy}

Ninety-five patients had received VEGF-targeted agents; 20 were still on treatment at the time of this analysis and the remaining 75 had stopped therapy for progression $(n=59)$, toxicity $(n=14)$ or other reason $(n=2)$. TTF on first VEGF-targeted therapy by mutation status is summarized in Table 3. TTF with first VEGF-targeted therapy differed significantly by $P B R M 1$ mutation status, where presence of acquired somatic mutation associated with more favorable TTF ( $p=0.01$; median 12.0 months vs. 6.9 months for WT). BAP1 mutation status correlated adversely with TTF in VEGF-targeted therapy treated patients ( $p=0.01$; median 6.4 months for MT vs. 11.0 months for WT). We found no significant correlation for patients harboring mutations in VHL, SETD2, $T E R T$, and $K D M 5 C$.

\section{DISCUSSION}

With tumor genomic profiling increasingly being used in the diagnostic workup of RCC patients, knowledge of mutation spectrum has grown, but prior studies were largely limited to nonmetastatic ccRCC [13-15]. This report represents an effort to correlate genomic profiling and outcomes with VEGF-targeted therapy in metastatic ccRCC patients. 


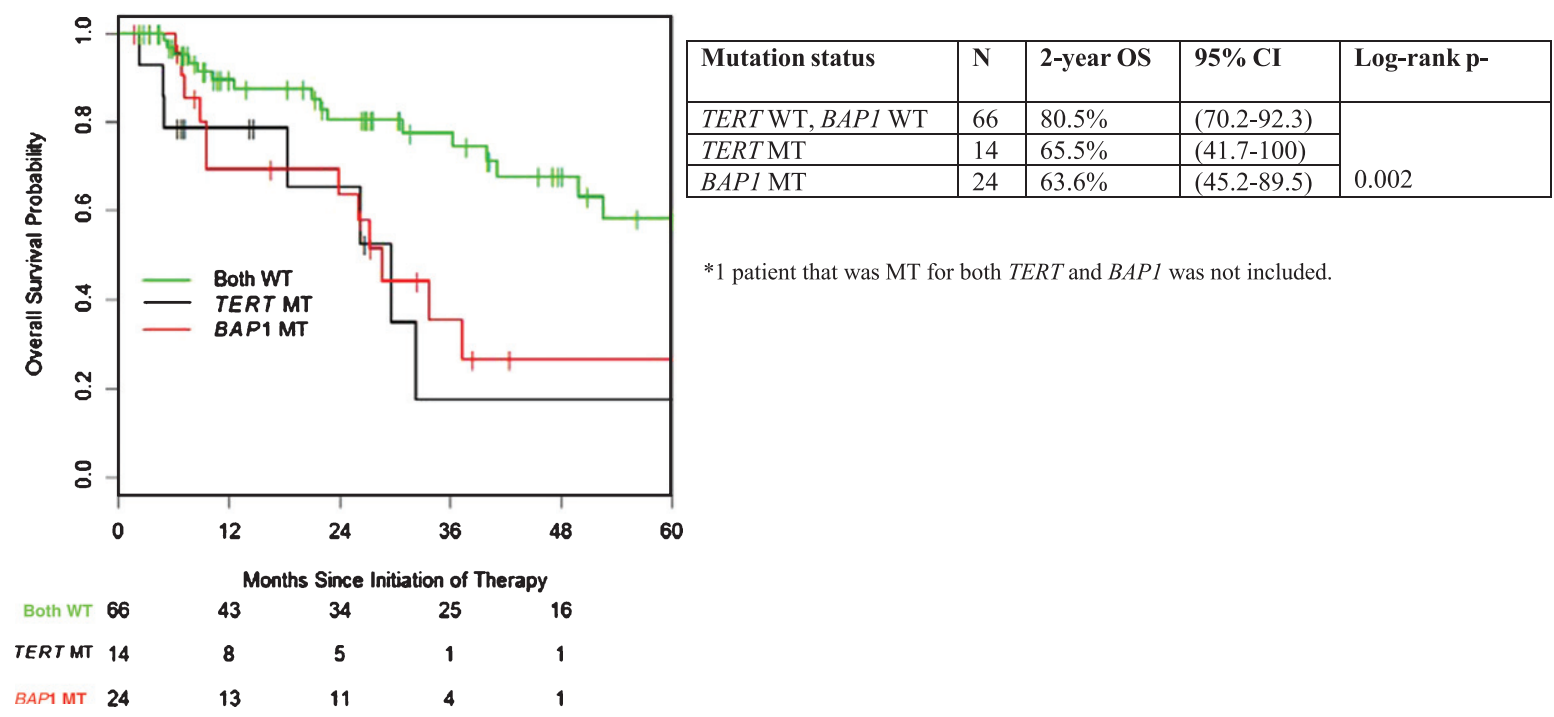

Fig. 2. Overall survival stratified by presence of TERT promoter and BAPl mutation.

Table 3

Time to failure (TTF) on first VEGF-targeted therapy by mutation status

\begin{tabular}{lcccc}
\hline Mutation status & $\mathrm{N}$ & $\begin{array}{c}\text { Median TTF } \\
\text { (months) }\end{array}$ & $95 \%$ CI & $\begin{array}{c}\text { Log-rank } \\
p \text {-value }\end{array}$ \\
\hline VHL WT & 16 & 6.5 & $(5.8-\mathrm{NA})$ & 0.82 \\
VHL MT & 79 & 10.6 & $(7.4-16.6)$ & \\
PBRM1 WT & 48 & 6.9 & $(4.6-10.8)$ & 0.02 \\
PBRM1 MT & 47 & 12.0 & $(10.0-21.6)$ & \\
SETD2 WT & 61 & 8.6 & $(6.7-12.2)$ & 0.72 \\
SETD2 MT & 34 & 10.0 & $(6.5-19.5)$ & \\
BAP1 WT & 73 & 11.0 & $(8.4-19.3)$ & 0.01 \\
BAP1 MT & 22 & 6.4 & $(3.9-16.6)$ & \\
KDM5C WT & 78 & 8.6 & $(6.5-12.2)$ & 0.90 \\
KDM5C MT & 17 & 11.4 & $(9.7-24.3)$ & \\
TERT WT & 82 & 10.6 & $(7.1-12.3)$ & 0.25 \\
TERT MT & 13 & 6.9 & $(2.3-\mathrm{NA})$ & \\
\hline
\end{tabular}

${ }^{*}$ MT, mutant; WT, wild-type; NA, not applicable.

In this cohort, recurrent alterations were detected across genes previously implicated in early stage disease, including VHL, PBRM1, SETD2, and BAP1, but at a higher frequency than in prior reports. For example, as compared to results from The Cancer Genome Atlas (TCGA), PBRM1 mutations were identified in 51 vs. $33 \%$, SETD2 in 37 vs. $12 \%$, and BAP1 in 24 vs. $10 \%$; this finding supports their suggested significance in the pathogenesis of this disease, including development of a metastatic phenotype [12]. Furthermore, recurrent alterations were detected in TP53 (11\%), and the TERT promoter region (13\%), which were not reported as commonly altered genes in earlier non-metastatic datasets. These may represent late events in the evolution of metastatic disease.
The presence of BAPl or TERT promoter mutations was associated with a significantly worse OS. These two mutations were mostly mutually exclusive, with only one tumor harboring mutations in both genes. BAPl codes for a deubiquitinating enzyme involved in chromatin remodeling, and several studies in mostly localized RCC have found that loss of $B A P l$ is associated with poor prognosis $[13,14]$. Although TERT mutations were not described in the original TCGA study of ccRCC, a recent study of mostly non-metastatic cases identified approximately $10 \%$ prevalence in ccRCC tumors, and also showed that there may be an association with aggressive disease [17]. TERT promoter mutations are the most common somatic noncoding mutations in cancer, and have also been associated with adverse outcomes in several malignancies, including melanoma, thyroid cancer, and bladder cancer [18-24]. An exploratory analysis was performed to investigate if acquired mutations in BAPI or TERT were associated with MSKCC prognostic risk group or the presence of sarcomatoid features on histopathologic review. While there was no association with mutation status and risk group, there was a significantly higher rate of sarcomatoid features in MT vs. WT $(p=0.008)$. Due to sample size constraints, we were unable to investigate whether these are independent risk factors for adverse outcomes; this should be analyzed in larger cohorts. Patients whose tumors displayed sarcomatoid features had worse overall survival regardless of mutation status, but among those without sarcomatoid features, BAPl or TERT MT had worse 
OS than WT (Supplemental Figure 1; Supplemental Table 3).

In this study, mutations in PBRM1 correlated with statistically longer TTF with VEGF-targeted therapy, with a trend towards superior OS (not reached for MT vs. 36.3 months for WT; $p=0.12$ ). Similar findings were previously reported for metastatic patients treated on the RECORD-3 trial, with a trend towards superior outcome for patients with PBRMI MT tumors, and in a recent analysis of ccRCC patients with extreme responses to VEGF-targeted therapy. In contrast to these findings in metastatic disease, early-stage studies have reported adverse association with cancer-specific outcome [14]. PBRM1 is a component of the SWI/SNF chromatin remodeling complex, and mutations of $P B R M 1$ are an early event in ccRCC tumor development $[25,26]$. Together, these findings may suggest that loss of PBRM1 is biologically relevant for invasiveness and metastatic spread; but once tumor cells metastasize, those with loss of PBRM1 may behave less aggressively than tumors which metastasize by other molecular means (e.g. mutations in BAPl or TERT) [14, 25, 27]. Such observations are limited by sample size, and without a control arm, it is not possible to determine whether PBRM1 mutation status is a predictive or prognostic biomarker.

In the prior analysis of the RECORD-3 trial, there was a significant correlation between presence of KDM5C mutation and PFS in 111 patients receiving sunitinib (median PFS 8.3 for KDM5C WT vs. 20.6 months for MT; $p=0.03$ ), but not for 109 everolimustreated patients (median PFS 8.2 for KDM5C WT vs. 9.8 months for MT; $p=0.03$ ). In the present analysis, TTF did not correlate significantly with KDM5C mutation status across 95 VEGF-targeted therapy treated patients (TTF 8.6 months for KDM5C WT vs. 11.4 months for MT; $p=0.9$ ). The inability to confirm the previously suggested correlation may relate to sample size and a more heterogeneous patient population.

This study has several other limitations. As a single-institution experience of limited sample size, findings will need to be corroborated in larger independent datasets. In addition, TTF, which is subject to individual decision making of treating physicians, was used as a measure of therapeutic benefit and limits comparison to more rigid assessment approaches, such as Response Evaluation Criteria in Solid Tumors (RECIST) [28]. Archival samples were derived from both primary tumor and metastatic sites, some having been collected long before the initiation of targeted therapy. Finally, intratumor heterogeneity is a well-known phenomenon in RCC and limits our accuracy in assessing the true mutant allele frequency in each sample [25, 29]. Nevertheless, biology of metastatic sites should guide survival for most patients, and recent data shows a high correlation of mutation profiles in primary vs. metastatic sites [30].

The data presented here cannot inform the choice of agent for patients with metastatic ccRCC, although the ever expanding number of approved targeted therapies leaves prescribers with a clear need for predictive biomarkers in this space. Our findings can, however, help better inform our understanding of pathobiology and clinical course of this disease. Certain genetic alterations, such as BAPl and TERT promoter mutations, may be present in higher frequency than previously thought and, based on this data, deserve further study for their prognostic implications. Ultimately, they may prove useful in refining our current standards of assessing risk status in metastatic disease, and could inform surveillance strategies and future trial design [31].

\section{ACKNOWLEDGMENTS}

We gratefully acknowledge the members of the Molecular Diagnostics Service in MSKCC's Department of Pathology.

\section{FUNDING}

This work was funded in part through the MarieJosée and Henry R. Kravis Center for Molecular Oncology and National Institutes of Health/National Cancer Institute Cancer Center Support (Grant P30 CA008748), Ruth L. Kirschstein National Research Service Award T32CA082088, Clinical and Translational Science Center at Weill Cornell Medical Center UL1TR00457 and the Randall MacDonald Kidney Cancer Research Fund. MIC is supported through a Kidney Cancer Association Young Investigator Award.

\section{DISCLOSURES}

MHV reports consulting fees (Pfizer, Eisai, Novartis, Exelixis, Calithera, Natera), research grants (BMS, Genentech/Roche) and travel funding (Novartis and Takeda). DRF reports consulting fees (Bayer and Seattle 75 Genetics) and research funding 
(Novartis). RJM reports consulting (Pfizer, Novartis, Exelixis, Eisai) and funding to MSKCC for clinical trial support (Pfizer, Genentech/Roche, Novartis, BMS, Eisai, and Exelixis). WL is employed by Helix, a for-profit company. CHL receives funding to MSKCC for clinical trial support (Pfizer and Eisai), and consulting (Exelixis). JJH reports honoraria (Chugai Pharmaceutical), consulting (Novartis, Chugai Pharmaceutical, Eisai) and research funding (Novartis, Pfizer, CGI). All the remaining authors have no disclosures.

\section{SUPPLEMENTARY MATERIAL}

The supplementary table and figure are available in the electronic version of this article: http://dx.doi. org/10.3233/KCA-160003.

\section{REFERENCES}

[1] Patard JJ, Leray E, Rioux-Leclercq N, et al. Prognostic value of histologic subtypes in renal cell carcinoma: A multicenter experience. J Clin Oncol 2005;23:2763-71.

[2] Siegel RL, Miller KD, Jemal A. Cancer statistics, 2016. CA Cancer J Clin 2016;66:7-30.

[3] Motzer RJ, Hutson TE, Tomczak P, et al. Sunitinib versus interferon alfa in metastatic renal-cell carcinoma. N Engl J Med 2007;356:115-24.

[4] Hudes G, Carducci M, Tomczak P, et al. Temsirolimus, interferon alfa, or both for advanced renal-cell carcinoma. NEngl J Med 2007;356:2271-81.

[5] Choueiri TK, Escudier B, Powles T, et al. Cabozantinib versus everolimus in advanced renal-cell carcinoma. New England Journal of Medicine 2015;373:1814-23.

[6] Motzer RJ, Hutson TE, Glen $\mathrm{H}$, et al. Lenvatinib, everolimus, and the combination in patients with metastatic renal cell carcinoma: A randomised, phase 2, open-label, multicentre trial. The Lancet Oncology 16:1473-82.

[7] Motzer RJ, Escudier B, McDermott DF, et al. Nivolumab versus everolimus in advanced renal-cell carcinoma. NEngl J Med 2015;373:1803-13.

[8] Chapman PB, Hauschild A, Robert C, et al. Improved survival with vemurafenib in melanoma with BRAF V600E mutation. New England Journal of Medicine 2011; 364:2507-16.

[9] Shaw AT, Kim DW, Nakagawa K, et al. Crizotinib versus chemotherapy in advanced ALK-positive lung cancer. N Engl J Med 2013;368:2385-94.

[10] Mok TS, Wu YL, Thongprasert S, et al. Gefitinib or carboplatin-paclitaxel in pulmonary adenocarcinoma. N Engl J Med 2009;361:947-57.

[11] Sato Y, Yoshizato T, Shiraishi Y, et al. Integrated molecular analysis of clear-cell renal cell carcinoma. Nat Genet 2013;45:860-7.

[12] Comprehensive molecular characterization of clear cell renal cell carcinoma. Nature 2013;499:43-9.

[13] Hakimi AA, Ostrovnaya I, Reva B, et al. Adverse outcomes in clear cell renal cell carcinoma with mutations of 3 p21 epi- genetic regulators BAP1 and SETD2: A report by MSKCC and the KIRC TCGA research network. Clin Cancer Res 2013;19:3259-67.

[14] Joseph RW, Kapur P, Serie DJ, et al. Clear cell renal cell carcinoma subtypes identified by BAP1 and PBRM1 expression. J Urol 2016;195:180-7.

[15] Kapur P, Pena-Llopis S, Christie A, et al. Effects on survival of BAP1 and PBRM1 mutations in sporadic clear-cell renalcell carcinoma: A retrospective analysis with independent validation. Lancet Oncol 2013;14:159-67.

[16] Cheng DT, Mitchell TN, Zehir A, et al. Memorial sloan kettering-integrated mutation profiling of actionable cancer targets (MSK-IMPACT): A hybridization capture-based next-generation sequencing clinical assay for solid tumor molecular oncology. J Mol Diagn 2015;17:251-64.

[17] Wang K, Liu T, Liu L, et al. TERT promoter mutations in renal cell carcinomas and upper tract urothelial carcinomas. Oncotarget 2014;5:1829-36.

[18] Vinagre J, Almeida A, Populo H, et al. Frequency of TERT promoter mutations in human cancers. Nat Commun 2013;4:2185

[19] Bell RJ, Rube HT, Xavier-Magalhaes A, et al. Understanding TERT promoter mutations: A common path to immortality. Mol Cancer Res 2016;14:315-23.

[20] Huang FW, Bielski CM, Rinne ML, et al. TERT promoter mutations and monoallelic activation of TERT in cancer. Oncogenesis 2015;4:e176.

[21] Melo M, da Rocha AG, Vinagre J, et al. TERT promoter mutations are a major indicator of poor outcome in differentiated thyroid carcinomas. J Clin Endocrinol Metab 2014;99:E754-65.

[22] Rachakonda PS, Hosen I, de Verdier PJ, et al. TERT promoter mutations in bladder cancer affect patient survival and disease recurrence through modification by a common polymorphism. Proc Natl Acad Sci U S A 2013;110:1742631.

[23] Qu Y, Dang S, Wu K, et al. TERT promoter mutations predict worse survival in laryngeal cancer patients. Int J Cancer 2014;135:1008-10.

[24] Griewank KG, Murali R, Puig-Butille JA, et al. TERT promoter mutation status as an independent prognostic factor in cutaneous melanoma. J Natl Cancer Inst 2014;106.

[25] Gerlinger M, Rowan AJ, Horswell S, et al. Intratumor heterogeneity and branched evolution revealed by multiregion sequencing. New England Journal of Medicine 2012;366:883-92.

[26] Varela I, Tarpey P, Raine K, et al. Exome sequencing identifies frequent mutation of the SWI/SNF complex gene PBRM1 in renal carcinoma. Nature 2011;469: 539-42.

[27] Gerlinger M, Horswell S, Larkin J, et al. Genomic architecture and evolution of clear cell renal cell carcinomas defined by multiregion sequencing. Nat Genet 2014;46: 225-33.

[28] Eisenhauer EA, Therasse P, Bogaerts J, et al. New response evaluation criteria in solid tumours: Revised RECIST guideline (version 1.1). Eur J Cancer 2009;45:228-47.

[29] Sankin A, Hakimi AA, Mikkilineni N, et al. The impact of genetic heterogeneity on biomarker development in kidney cancer assessed by multiregional sampling. Cancer Med 2014;3:1485-92.

[30] Guillermo de Velasco RM, Mahamed Ali S, Signoretti S, Mullane SA, Ross JS, Miller VA, Stephens PJ, Schrock AB, Young L, Pal SK, Choueiri TK. Genomic profiling of 
nephrectomy and metastatic sites in patients with advanced clear cell renal cell carcinoma (RCC) [abstract]. In Genitourinary Cancers Symposium 2017.

[31] Rini BI, Dorff TB, Elson P, et al. Active surveillance in metastatic renal-cell carcinoma: A prospective, phase 2 trial. The Lancet Oncology 17:1317-24.
[32] Feldman DR, Baum MS, Ginsberg MS, et al. Phase I trial of bevacizumab plus escalated doses of sunitinib in patients with metastatic renal cell carcinoma. J Clin Oncol 2009;27:1432-9. 